\title{
Boredom among psychiatric in-patients: does it matter?
}

\author{
Rachel Steele, Paul Henderson, Frances Lennon \& Donna Swinden
}

\begin{abstract}
SUMMARY
Many psychiatric in-patients report boredom. Such complaints may appear trivial, but this literature review by a clinical librarian suggests that boredom is more complex than may initially appear and relates to wider areas of importance for in-patient psychiatric teams. Boredom may relate to the internal experience of meaning, which itself encompasses meaningful relationships and roles and a sense of control. Although meaningful therapeutic activities are vital, mental health professionals should focus on the internal as well as the external dimensions of boredom. Medications, particularly dopamine antagonist antipsychotics, may be a contributing factor. This article highlights the benefits of the clinical librarian role in synthesising research in mental health. Research can illuminate psychiatric practice in a more holistic way than purely by applying 'scientific' evidence in the practice of 'biological' psychiatry.
\end{abstract}

\section{DECLARATION OF INTEREST}

None.

This critical literature review on boredom arose out of a pilot clinical librarian/informationist project in Tees, Esk and Wear Valleys (TEWV) NHS Foundation Trust. At the time of writing, the lead author (R.S.) was clinical librarian/informationist for four pilot teams in the trust. One of these teams includes a consultant psychiatrist (P.H.), a modern matron (D.S.) and an occupational therapist (F.L.) in an adult acute in-patient service. The consultant psychiatrist suggested that R.S. consult the research evidence base to illuminate in-patients' reports of 'boredom'. The body of this article, including the conclusions, has been written by R.S., with commentaries by P.H., F.L. and D.S.

\section{Is in-patient boredom important?}

Boredom is a topic that has received little empirical attention in clinical settings (Goldberg 2011). Although patients' complaints that they are 'bored' may sound trivial, there is some (although limited) evidence that boredom is associated with a range of illnesses and dysfunctional behaviour, for example as a trigger for relapse in dual diagnosis
(Laudet 2004), in substance misuse more generally (Corvinelli 2005) and in absconding from psychiatric hospitals (Bowers 1999). Although the possible association between boredom and psychiatric disorders is complex, Newell et al (2012) found a correlation between depression and boredom, and Todman (2003) argues that boredom may be a prodromal marker in psychosis. The experience of boredom therefore has much wider implications than may initially appear.

\section{Why ask a clinical librarian?}

As this article is one of many outputs from the TEWV Clinical Librarian Project, it is appropriate to highlight briefly the rationale for the initiative. Clinical librarians have been in existence for around 40 years. They provide research summaries to support clinical decision-making and to inform strategy and changes.

There have been very few clinical librarian projects in mental health. A notable exception is a study by Gorring et al (2010), which concluded that the clinical librarian role can be effective in implementing evidence-based psychiatric practice in clinical teams.

Davidoff $\&$ Florance (2000) acknowledge the contributions that clinical librarians can make in stimulating access to the evidence base, pointing out that many now read the literature and write brief synopses of papers to present on ward rounds and at conferences.

During the TEWV Clinical Librarian Project, it soon became apparent that clinicians valued the role of an information specialist/librarian who could critically appraise and synthesise research, as well as the more traditional role of the healthcare librarian, which focuses on information searching.

\section{Benefits and uses of evidence syntheses in mental health}

This article is an instructive example to consultant psychiatrists of the benefits of the clinical librarian's evidence-synthesising role, and consequently of research evidence synthesis, to mental healthcare.
ARTICLE

Rachel Steele is a clinical librarian with Tees, Esk and Wear Valleys NHS Foundation Trust. Her main interests include the emerging role of the clinical librarian/ informationist in leading and facilitating access to the evidence base in all aspects of mental health services, as well as educational/ CPD interventions to develop health professionals' skills in using evidence in their practice. Paul Henderson is a consultant psychiatrist, Frances Lennon is an occupational therapist and Donna Swinden is a modern matron, all based at the time of writing in an adult acute in-patient service of Tees, Esk and Wear Valleys NHS Foundation Trust. Correspondence Rachel Steele, Library and Information Service, Education Centre, Lanchester Road Hospital, Durham DH1 5RD, UK. Email: rachelsteele@nhs.net 
The argument that evidence-based medicine is not restricted to randomised trials and metaanalyses is not new (see Sackett 1996), and the National Institute for Health and Care Excellence (as NICE is now named) believes that all practice, not just scientific approaches, should be evidence based. Nevertheless, there is anecdotal evidence that evidence-based practice is seen as more relevant for 'biological' psychiatry than for the more subtle, greyer areas of clinical practice (Cooper 2003; Hannes 2010; Jainer 2011). These 'greyer' areas might include a myriad of factors relating to the holistic patient/service user experience and to questions of diagnosis, prognosis and aetiology: factors that do not so easily lend themselves to the collection of scientific (particularly randomised controlled trial, RCT) evidence in assessing the efficacy of treatments (particularly drug interventions) more commonly associated with evidence-based practice.

Geddes et al (1996) found that the majority of primary interventions for psychiatric inpatients were based on 'high-level' experimental evidence. They did, however, note that most such interventions were pharmacological and that RCT evidence is more likely to be available in this area than for non-pharmacological approaches. However, our study shows how non-RCT evidence (mainly qualitative research, cross-sectional studies, case studies and a literature review) can also have an impact on holistic patient care and services. The study's search strategy is outlined in Box 1.

\section{B0X 1 The boredom search strategy}

R.S. searched bibliographic databases (AMED, BNI, CINAHL, the Cochrane Library, Embase, MEDLINE and PsycINFO) using 'boredom' and synonymous keywords (e.g. 'monotony') and search strings relating to in-patient psychiatry, such as (ward* OR inpatient ${ }^{*}$ OR in-patient ${ }^{*}$ OR hospital ${ }^{*}$ OR acute) AND (psych* OR mental*). Database thesaurus terms relating to boredom or in-patient psychiatry were combined with keyword searches.

Supplementary literature searches included keywords and thesaurus terms relating to therapeutic groups in in-patient psychiatry, the third (voluntary) sector providing inreach into psychiatric hospitals, in-patient 'hearing-voices' approaches, and medications and boredom. References identified by electronic searching were consulted.
The challenge in setting inclusion and exclusion criteria was to provide a broad enough perspective to give a useful overview but to be related enough to inpatient psychiatry to be of practical use. The initial inclusion criteria were that papers had to consider boredom in its entirety in the in-patient context. The review was then widened to cover boredom and adult mental illness more globally (although excluding older adult populations), because a tight focus on the in-patient setting omits theoretical frameworks which can illuminate broader understanding

There is a separate evidence base which has considered leisure or occupational boredom, but this was not included in the study.

\section{What is boredom?}

Patients on many psychiatric wards report boredom (National Institute for Health and Clinical Excellence 2011). The Royal College of Psychiatrists (2011: p. 10) states that boredom impedes recovery and can lead to unsafe and violent behaviour. The College has explicitly linked the 'therapeutic ward' and structured activities to reduction of patient boredom, arguing that all staff, not just occupational therapists, should consider keeping patients engaged 'as important a function as administering medication’ (p. 12).

\section{Situation-dependent and situation-independent boredom}

The notion that boredom is due to inadequate outside stimulation is intuitively appealing. As Newell (2009) argues, 'The general consensus in psychology and occupational science literature is that boredom is a drive of low arousal' (p. 25). There are two major, non-mutually exclusive, conceptions of boredom - situation-dependent boredom and situation-independent boredom (Todman 2003). Proponents of situation-dependent boredom believe that boredom is caused by monotonous environmental conditions, that once these are removed, boredom will be alleviated, and that individual differences in boredom reflect a differing level of vulnerability to environmental stimuli, with 'high-risk' individuals employing certain (usually maladaptive) behaviours or traits. In contrast, proponents of situation-independent boredom (who tend to be psychoanalytically oriented or existential theorists) argue that boredom 'results from an inability to utilize the coping mechanisms needed to provide escape from monotony [...] (i.e. fantasy, anticipation, etc.)', which is independent of environmental conditions (Todman 2003: p. 149).

Situation-dependent boredom is therefore a view of boredom as 'externally' driven, whereas situation-independent boredom emphasises 'internal' factors. There is no clear consensus on whether situation-dependent or situationindependent theories of boredom are more accurate. The bulk of our article, however, follows Binnema's (2004) 'internal', sociological view of boredom because his theoretical proposition is more closely based on the psychiatric in-patient population than most other research reports. However, in a wider sense it is helpful, as Newell (2009: p. 25) believes, to recognise that sources of stimulation can be either internal or external to the individual, and therefore that there are internal and external components of boredom. 
Boredom is therefore more complex than simply 'not having enough to do.'

\section{The 'internal' experience of meaning and boredom}

\section{Boredom and the experience of meaning}

A key piece of evidence in relation to psychiatric patients' experiences of boredom is Binnema's (2004) literature review. Binnema argues that boredom is an emotion interconnected with the experience of meaning. It should be noted that this internally driven theory is one of several competing theories of boredom. According to Binnema's view, complaints of boredom represent patients' emotional response to a decreased sense of meaning. The notion that boredom is related to 'life meaning' (i.e. having a purpose in life) is supported by Fahlman (2009), who found that boredom and life meaning share a closer relationship with each other than with depression or anxiety in undergraduates.

Binnema identifies three interrelated concepts which he argues are central to the experience of meaning in life:

- being in meaningful relationships

- having meaningful roles

- having a sense of control.

The centrality of these dimensions to the experience of meaning is highlighted in Box 2, in which a service user links participation in therapeutic groups to decreased stigma and promotion of social inclusion. This individual also found mindfulness helpful, which ties in with another internally driven theory of boredom currently gaining interest within cognitive neuroscience that boredom is associated with inattention.

Eastwood et al (2012) believe that boredom should be defined in terms of attention, and Malkovsky et al (2012) have explored the relationship between sustained attention and proneness to boredom. The Malkovsky team identify two types of boredom: apathetic, where the person is unconcerned with their environment; and agitated, where the person tries to engage in meaningful activities but these fail to satisfy. In their study of undergraduates, the team found that proneness to apathetic boredom was associated with attention lapses, and proneness to agitated boredom was associated with reduced sensitivity to errors of sustained attention and increased symptoms of adult attention-deficit hyperactivity disorder.

Martin (2008: p. 154) theorises that boredom is associated with a lack of mindfulness and argues that 'it might be possible to overcome a propensity to boredom through learning how to be mindful'.
BOX 2 A service user's experience of the therapeutic benefits of activitybased groups in in-patient units
'One of the main problems for people with mental health issues is social exclusion. Often met with stigma, our needs to develop and maintain jobs, relationships, interests and other roles can lead to rejection causing a breakdown in our daily lives. I believe that the activities provided by the hospital are essential tools in counterattacking this breakdown.

The opportunity to participate in activitybased groups in the hospital setting is the first step towards social inclusion. In my experience it has been the foundation to rebuilding my confidence and self-esteem. Physical activities have a phenomenal effect on our minds as well as our bodies
The Thai boxing classes and mindfulness sessions were exceptionally helpful to me. Thanks to the hospital I was able to use the Thai boxing classes to beat out the negativity stored up and replace it with positive thinking by applying mindfulness. I am truly grateful for the help and support from the activities team, who became a huge part of my treatment.

Since my discharge I have become a member at a mixed martial arts gym where I train in Brazilian ju-jitsu and Thai boxing. This is something I would never have done had it not been for the activities team introducing me to the sport and helping me to recognise my potential.'

(Published with permission of the service user)

\section{Being in meaningful relationships}

Binnema (2004) asserts that social relationships are empowering and help create experiences of meaning, but that psychiatric in-patients have few opportunities for meaningful social interactions. The significance of social relationships is supported by Thomas et al's (2002) qualitative study of eight in-patients. All of the participants thought that 'therapy' administered by their peers was the most beneficial aspect of their time in hospital, and they 'expressed longing for a deeper connection with staff'. Evidence has also been put forward by NICE that some in-patients feel they receive more support from fellow patients than staff (National Institute for Health and Clinical Excellence 2011).

Thomas et al's findings are supported by Higgins et al's (1999) study of acute psychiatric wards, which reported that nurses' activity was focused on a minority of severely ill patients, which significantly limited their opportunity to implement coordinated programmes of care for all patients. Although patients valued their named nurse's support, many had 'only passing relationships with nurses', were bored and 'felt they were often left to their own devices'.

Nevertheless, there are examples of healthcare professionals addressing in-patients' needs for meaningful relationships. In their report on inpatient nurses using Romme $\&$ Escher's approach to help voice-hearers develop a narrative account of their voices, Place et al (2011: p. 841) note the strong relationship that develops between nurse and patient. Ruddle et al (2011) argue that group 
treatment offers advantages over individual therapy for voice-hearers. Place et al's successful therapeutic partnership approach could therefore be replicated in 'hearing-voices groups' which could address in-patients' needs for meaningful relationships with staff and other patients.

\section{Having meaningful roles}

Binnema (2004: p. 839) argues that offering psychiatric patients the opportunity to take on meaningful roles while in hospital may give them a sense of meaning and therefore empowerment. Such roles could be provided through purposeful activities. Indeed, NICE stresses that psychiatric in-patients should have access to 'meaningful and culturally appropriate' activities (National Institute for Health and Clinical Excellence 2011: p. 118). Antoniou's (2007) experience as a service user supports Binnema's assertion that boredom may be linked to the absence of meaningful roles or roles that patients are used to when not in hospital. She writes:

"There are two sorts of "boring" on the ward. One is caused by having lots of hours to fill and not having the things one usually has to fill them up with because one is not at home [...] It is possible to be bored on the ward even when there are activities planned every hour, and not to be bored when doing nothing' (Antoniou 2007: p. 33).

This argument is potentially relevant to wardbased group activities. Simpson et al (2005) reported that some nurses, ward managers and psychiatrists saw the occupational therapist's role as largely focused on group activities: 'Often these were seen as predominantly diversionary or time-filling activities, with the more explicitly therapeutic functions [...] rarely acknowledged' (p. 547-8). Newell (2009: p. 26) believes that organised group activities deal only with external components of boredom, so the provision of more activities does not necessarily reduce complaints of boredom.

If activities do not provide opportunities to experience meaningful roles, then it is unlikely that they will exert their full therapeutic effect. Farnworth et al (2004), in a naturalistic inquiry study involving eight patients in an Australian secure forensic psychiatric unit, found that popular groups offered 'a specific purpose and outcome' or encompassed 'novelty, creativity or nonverbal components to performance' (p. 435). For example, the 'cook your own meal' group had a waiting list. However, many of the activities on offer seemed to lack 'novelty, challenge and personal meaning' and patients thought them boring or 'old hat' (p. 435).
Caddy et al (2011) reported that participation in creative activity groups positively correlated with improved mental health in an acute in-patient psychiatric hospital in Australia. However, they pointed out that not everyone finds such groups meaningful or beneficial, so group allocation should reflect the needs of individual patients (p. 331).

There may also be a role for partner organisations who could 'in-reach' to meet patients' needs for meaningful activities while in hospital and provide continuity of therapeutic input after discharge. There are limited examples of partner/ voluntary organisations working in this way, but Thomas et al (2012) report a successful voluntary activities programme that complements statutory child and adolescent mental health services. The service user whose experiences are described in Box 2 also highlights the benefits of continuing therapeutic activities pre- and post-discharge.

\section{Having a sense of control}

The need of patients to have a say in the activities and occupations offered on the wards has been recognised by NICE (National Institute for Health and Clinical Excellence 2011). Binnema (2004) likewise argues that 'Loss of control [...] is related to decreased mental health. Conversely, empowerment [...] and thus decreased boredom, is related to increased mental health' (p. 836). He acknowledges that loss of control is intrinsically related to the nature of psychiatric illnesses, but also argues that authoritarian psychiatric nursing may encourage patient dependency. The challenge is to empower patients by harnessing the potential of the hospital therapeutic environment.

Farnworth et al (2004) cite the example of a service user who was dissatisfied with ward occupations but felt that he had learnt how to gain control over his time. Interestingly, they note that he was the only participant interviewed who said that he was 'quite happy with [his] life' (p. 435).

If a lack of control is related to boredom, we might expect there to be an association between autonomous activities and reduced boredom. Indeed, Newell et al (2012) reported that, although there was an association between in-patients' engagement in autonomous activity and decreased self-reports of boredom, 'There was no evidence of any relationship between boredom proneness and organised activities' (p. 6).

Given the importance of autonomy, Newell et al (2012) hypothesised that involuntary inpatients would be more prone to boredom. However, their study indicated the opposite. A potential confounder was that more voluntary 
than involuntary patients were diagnosed with depression and 'depression is highly correlated with proneness to boredom'. We return to the complex relationship between depression and boredom in 'Associations between boredom and psychiatric diagnoses', below.

As numerous authors (e.g. Simpson 2005; Hitch 2009) argue, occupational therapists are often dismayed to receive referrals of patients who are 'bored', believing that this shows a limited understanding of their role. Shattell (2007) also notes that many nurses react negatively to patients' boredom, saying that their role is not 'to entertain patients'. However, Newell (2009: p. 27) believes that ultimately 'we should be helping our service users to address boredom for themselves'.

\section{Medication and boredom}

In examining boredom, it is difficult to disentangle the effects of medication from those of psychiatric illness, but there is some evidence that medication could be a contributing factor independent of psychiatric disorder. Healey (2009) notes that almost all antidepressants 'can be somewhat sedative' and that with selective serotonin reuptake inhibitors (SSRIs) 'there may be a paradoxical coexistence of feeling drowsy or fatigued' (p. 70). He also points out that the sedative effect of all benzodiazepines except clobazam is well documented (p. 154). If we consider theories of situation-dependent boredom, which stress the centrality of environmental conditions, then the sedative/attention-impairing effects of these medications could contribute to patients' experiences of environmental sameness and thus feelings of boredom. Internally driven theories positing a link between boredom and inattention may also be significant (Eastwood 2012; Malkovsky 2012). If medications induce sedation, and consequently impair attention or concentration, this may also be a contributor to boredom.

The antipsychotics are also a potentially significant contributor because of their blocking of the $\mathrm{D}_{2}$ dopamine receptor. The mesolimbic dopamine pathway is important for motivation, pleasure and reward, as well as the positive symptoms of psychosis (Stahl 2008: p. 272). Wise (2004) concludes that "brain dopamine seems to "stamp-in" response-reward and stimulus-reward associations that are essential for the control of motivated behaviour by past experience' ( $p$. 10). Flaherty (2011) also argues that 'Creativity depends on goal-driven approach motivation from midbrain dopaminergic systems' (p. 132).

It is difficult to determine the extent to which the dopamine antagonist effects of antipsychotics may contribute to a lack of drive, and consequently boredom, in people with schizophrenia because of the nature of the negative symptoms of the disorder. However, studies of healthy volunteers offer indications. Artaloytia et al (2006) reported that single doses of haloperidol, and particularly of risperidone, produced negative symptoms in healthy volunteers. Park et al (2012) likewise found that a single dose of risperidone induced negative symptoms (including increased avolition), although they concluded that haloperidol, amisulpride and aripiprazole did not.

It is therefore worth considering medication as a possible contributor to boredom when reviewing drug choice and dosage.

\section{Associations between boredom and psychiatric diagnoses}

Newell et al (2012) investigated proneness to boredom in a cross-sectional sample of 55 psychiatric in-patients. The study should be regarded as preliminary because of its design and small sample, but as far as we know it is the only study that has investigated the prevalence of boredom in adult psychiatric in-patients. The authors concluded that the three variables most predictive of boredom were having depression, anxiety or perhaps a personality disorder.

The possible association/relationship between boredom and psychiatric disorders is complex. Clinicians may mistake boredom for depression or negative symptoms of schizophrenia, but boredom does seem to be a distinct phenomenon. Goldberg et al (2011), in a cross-sectional sample of undergraduates, reported higher correlations between boredom and depression than between boredom and apathy or anhedonia, but found that depression and boredom were empirically distinct.

Todman (2003) argues, on the basis of clinical and non-clinical empirical studies and case studies of out-patients with psychosis, that boredom may be a prodromal marker in psychosis but that this may work in a heterogeneous and sometimes non-intuitive manner. He argues against the implicit assumption of the vulnerability-stresscoping model of schizophrenia, i.e. that external stimulation should be reduced. His reasoning is that, although some patients may be motivated to avoid excessive stimulation, others (especially in partially or fully remitted states) may be as motivated to avoid unstimulating environments as non-psychotic individuals. However, as this theoretical assertion is based on single cases (albeit with reference to prior research), further investigation is needed about the potential value of boredom as a prodromal marker in psychosis. 


\section{Implications for practice in adult acute in-patient services}

\section{Specialist commentary by Paul Henderson, consultant psychiatrist}

Before commenting on the clinical content of the article, I think it is important to highlight the TEWV Clinical Librarian Project in general. As a newly qualified consultant working in a busy general adult ward, the variety of presentations I see, the rapidity with which they present, and the multitude of pressures on my time mean that it has simply not been feasible to be as up to date across as wide a variety of topics as I would like. This has probably been the greatest source of professional and personal discomfort in my short consultant career thus far. As an addition to the multidisciplinary team (MDT), the clinical librarian has made a significant impact in this area, both for myself and the wider team, with junior colleagues, nursing staff, occupational therapists and psychologists all making use of the service she provides. When compared with all the other aspects of clinical governance the MDT practises, I have no doubt that the addition of the clinical librarian to the team has made the single largest improvement.

My motivation to ask the clinical librarian to provide an evidence summary on boredom was that I had become increasingly aware of the apparent dichotomy that ward staff appear to be constantly rushing, but many patients complain of being bored; and that, because of time pressures, my own practice was becoming more and more focused on the biological aspects of treatment. In the context of constant pressure to promote a more therapeutic environment, with a view to shorter hospital stays, it appeared to me that a broader perspective would be helpful.

While I am not suggesting that a sole focus on reducing patients' boredom is sufficient, or indeed necessary, the richness and nuances of the concepts in this article have certainly provided fresh and interesting ideas for the whole in-patient MDT to work on together. The insights into internal and external factors, as well as the probable superiority of autonomously driven activities, as opposed to generic group activities, have certainly significantly informed the ceaseless process of change that we are engaged in.

\section{Specialist commentary by Donna Swinden, modern matron}

The TEWV Clinical Librarian Project has been very beneficial to the clinical ward teams as it has enabled us to quickly access the evidence base for questions that arise during MDT discussions. The exploration of boredom is a particular example of this, as we have an extensive activity programme for our acute wards, with a variety of group and individual sessions, plus an on-site gymnasium. Our intensive support team also access community facilities, so we were rather puzzled when we often received negative feedback citing lack of activities. We regularly ask during patient ward meetings whether there are any other activities that patients would like to participate in, but we receive very few suggestions. This led us to consider a number of explanations - for example, lack of usual routine, that illness may prevent patients engaging in activities, or that some people will be bored whatever activities are on offer.

The evidence base produced by the clinical librarian confirms to some degree our suspicions and led to some useful discussions of how we might address the problem of boredom. It has prompted the team to make more use of the patient workbook so that patients can state their own interests and ideas of what would help them. We have also implemented a handover between nursing staff and intensive support staff every morning making use of the patient's workbook, so that intensive support staff are better informed as to what activities/interventions might be best for the individual on that particular day. Our intensive support team also monitor the uptake of activities, recording when patients have been offered an activity and have declined it. We are waiting to see whether our patient experience surveys will reflect these changes. We have also introduced more formalised protected therapeutic time so that patients can have more one-to-one time with the nursing team. We managed this by limiting MDT meetings to specific times of the day. This was introduced at the start of 2012 and is working well.

A further benefit of the TEWV Clinical Librarian Project is that the whole team, from nursing assistant through to consultant psychiatrist, become involved in discussions and generate ideas. This has enabled staff who do not traditionally access research, or who do not have the time to do so, to become familiar with literature reviews and experience their usefulness.

\section{Specialist commentary by Frances Lennon, occupational therapist}

We would not usually see our own activities as simply 'occupying' or 'filling in' time. We can be just as 'bored' when doing an activity that has no meaning or purpose to us as doing nothing at all. Occupational therapists aim to empower people 
to develop skills to plan their own time in a meaningful, individual way, and to transfer these skills into everyday life.

Occupational therapist assessment and treatment includes graded use of activities (i.e. facilitating activities at an appropriate level in the person's journey) to reduce and gain control of mental health symptoms and to support recovery. We work jointly with clients in developing their skills for life. Graded use of activities on leave can promote daily living skills and can counteract the reduced opportunity for usual routines when on the ward. Linking with community resources relevant to the individual can assist in the transition between hospital and home.

Therapeutic groups on and off the ward are aimed at helping people overcome mental health symptoms at each stage of being acutely unwell. These groups should not be characterised as 'give them something to do'. 'Doing' needs to be meaningful, purposeful and individually relevant. If it is not, the groups will only address external dimensions of boredom.

The TEWV Clinical Librarian Project has enabled a reflective step back to review what we do and how we do it, to confirm that something works, or to plan change.

\section{Conclusions}

\section{Boredom among in-patients}

Boredom among psychiatric in-patients is considerably more complex than simply a response to a lack of external stimulation that can best be tackled by diversionary activities. Boredom relates to the experience of meaning, which itself encompasses relationships, roles and a sense of control. Furthermore, medication, particularly antipsychotics that block dopamine (which is important for experiencing motivation, pleasure and reward), may contribute to it.

Meaningful therapeutic activities are vital, but mental health professionals should be aware of the internal and external dimensions of boredom. These can be linked, for example by a recognition that activities (external) are important, but are more likely to be successful if patients' needs for autonomy (internal) and meaning (internal) are met. Box 3 gives some practical suggestions for alleviating boredom on the ward. Box 4 outlines the key clinical learning points of this article, which have implications for multidisciplinary adult acute in-patient ward teams.

\section{Further research}

The possible association between boredom and psychiatric disorders is complex, but it does seem

B0X 3 Team approaches to alleviating boredom

Internal aspects of boredom

- Use a patient workbook to coordinate a personalised activity plan: meaningful activities can help patients experience meaningful roles

- Use the workbooks daily to plan individual and group activities (and involve all staff groups)

- Monitor the uptake of activities and review patients' views on relevance and satisfaction

- Protect one-to-one therapeutic time with patients by reducing meetings and administration

- Regularly review potential negative effects of medication on motivation and concentration
- Use Romme \& Escher's voice-hearing approach to provide meaningful therapeutic interventions for individual patients who hear voices

- Facilitate peer networks

External aspects of boredom

- Offer popular groups that are meaningful to individuals

- Use a graded approach to activities (i.e. facilitate activities at an appropriate level in the patient's journey)

- Use Romme \& Escher's voice-hearing approach to facilitate therapeutic groups for voice-hearers

- Partner with third-sector (voluntary-sector) groups to increase the variety of activities available from the ward

that boredom as a phenomenon distinct from psychiatric illness is worth researching. Further original research (particularly with a longitudinal design and larger samples) is needed to investigate boredom on adult psychiatric wards. It would be especially helpful if studies could investigate: associations/relationships between boredom and psychiatric diagnosis; the efficacy of psychosocial interventions designed to alleviate in-patients' experiences of boredom; and the extent to which medications (particularly dopamine antagonist antipsychotics) may be a contributor to boredom,

BOX 4 Key learning points for promoting a therapeutic environment that addresses patients' boredom in a holistic way

- Be aware that there are internal and external dimensions to boredom. Boredom may be due to a lack of stimulation (external) but is also likely to be caused by a lack of experience of meaning (internal)

- 'Internal' and 'external' approaches to the treatment of boredom can be combined, for example by recognising that (external) activities are important but they should be (internally) meaningful for patients and autonomously driven

- The lack of experience of meaning is likely to encompass three dimensions: the experience of being in meaningful relationships

the experience of being in meaningful roles the experience of having a sense of control

- Generic 'diversionary' activities (either individual or group based) are likely to address only the external dimensions of boredom, so may not provide long-lasting solutions

- All staff, not just occupational therapists, should work together to maintain a therapeutic environment to keep patients engaged and alleviate boredom

- Medications (particularly antipsychotics that block dopamine) may contribute to boredom

- Clinicians may mistake boredom for depression or negative symptoms of schizophrenia 


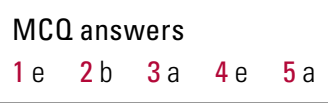

independent of the psychiatric conditions they are designed to treat.

\section{The role of clinical librarian and the complexity of 'evidence'}

By developing the role of the clinical librarian as evidence synthesiser, the full potential of 'evidence' (which uses a multiplicity of research designs, not just 'scientific' approaches) to illuminate clinical practice can be realised.

\section{Acknowledgements}

R.S. would like to thank Catherine Ebenezer and Bryan O'Leary for their support and encouragement in the writing of this article. All four authors thank the service user who kindly provided a patient perspective in Box 2, and Advances peer reviewers for their valuable comments.

\section{References}

Antoniou J (2007) Bored on the ward. In Experiences of Mental Health In-Patient Care: Narratives from Service Users, Carers and Professionals (eds M Hardcastle, D Kennard, S Grandison, et al): 33-40. Routledge.

Artaloytia JF, Arango C, Lahti A, et al (2006) Negative signs and symptoms secondary to antipsychotics: a double-blind, randomized trial of a single dose of placebo, haloperidol, and risperidone in healthy volunteers. American Journal of Psychiatry 163: 488-93.

Binnema DJ (2004) Interrelations of psychiatric patient experiences of boredom and mental health. Issues in Mental Health Nursing 25: 833-42.

Bowers L, Jarrett M, Clark N, et al (1999) Absconding: why patients leave. Journal of Psychiatric and Mental Health Nursing 6: 199-205.

Caddy L, Crawford F, Page AC (2011) 'Painting a path to wellness': correlations between participating in a creative activity group and improved measured mental health outcomes. Journal of Psychiatric and Mental Health Nursing 19: 327-33.

Cooper B (2003) Evidence-based mental health policy: a critical appraisal. British Journal of Psychiatry 183: 105-13.

Corvinelli A (2005) Alleviating boredom in adult males recovering from substance use disorder. Occupational Therapy in Mental Health 21: 1-11.

Davidoff F, Florance V (2000) The informationist: a new health professional? Annals of Internal Medicine 132: 996-8.

Eastwood J, Frischen A, Fenske M, et al (2012) The unengaged mind: defining boredom in terms of attention. Perspectives on Psychological Science 7: 482-95.

Fahlman SA, Mercer KB, Gaskovski P, et al (2009) Does a lack of life meaning cause boredom? Results from psychometric, longitudinal, and experimental analyses. Journal of Social and Clinical Psychology 28 : $307-40$.

Farnworth L, Nikitin L, Fossey E (2004) Being in a secure forensic psychiatric unit: every day is the same, killing time or making the most of it. British Journal of Occupational Therapy 67: 430-8.

Flaherty AW (2011) Brain illness and creativity: mechanisms and treatment risks. Canadian Journal of Psychiatry 56: 132-43.

Geddes J, Game D, Jenkins N, et al (1996) What proportion of primary psychiatric interventions are based on evidence from randomised controlled trials? Quality in Health Care 5: 215-7.

Goldberg YK, Eastwood JD, Laguardia J, et al (2011) Boredom: an emotional experience distinct from apathy, anhedonia or depression. Journal of Social and Clinical Psychology 30: 647-66.

Gorring H, Turner E, Day E, et al (2010) A clinical librarian pilot project in psychiatry. Psychiatrist 34: 65-8.
Hannes K, Pieters G, Goedhuys J, et al (2010) Exploring barriers to the implementation of evidence-based practice in psychiatry to inform health policy: a focus group based study. Community Mental Health Journal 46: 423-32.

Healey D (2009) Psychiatric Drugs Explained. Churchill Livingstone.

Higgins R, Hurst K, Wistow G (1999) Nursing acute psychiatric patients: a quantitative and qualitative study. Journal of Advanced Nursing 25: 833-42.

Hitch T (2009) Thoughts on... Boredom, mental health inpatients and occupational therapy by Sue Newell. Mental Health Occupational Therapy 14: 58.

Jainer AK, Teelukdharry SR, Onalaja D, et al (2011) Understanding the obstacles to evidence based practice in psychiatry: qualitative study of staff perceptions. International Medical Journal 18: 163-6.

Laudet AB, Magura S, Vogel HS, et al (2004) Perceived reasons for substance misuse among persons with a psychiatric disorder. American Journal of Orthopsychiatry 74: 365-75.

Malkovsky E, Merrifield C, Goldberg Y, et al (2012) Exploring the relationship between boredom and sustained attention. Experimental Brain Research 221: 59-67.

Martin M (2008) The phenomenon of boredom and its relationship to mindfulness. British Journal of Occupational Therapy 71: 154.

National Institute for Health and Clinical Excellence (2011) Service User Experience in Adult Mental Health: Improving the Experience of Care for People Using Adult NHS Mental Health Services (NICE Clinical Guideline 136). NICE.

Newell S (2009) Boredom, mental health inpatients and occupational therapy. Mental Health Occupational Therapy 14: 25-7.

Newell SE, Harries P, Ayers S (2012) Boredom proneness in a psychiatric inpatient population. International Journal of Social Psychiatry 58: 488-95

Park CH, Park TW, Yang JC, et al (2012) No negative symptoms in healthy volunteers after single doses of amisulpride, aripiprazole, and haloperidol: a double-blinded placebo-controlled trial. International Clinical Psychopharmacology 27: 114-20.

Place C, Foxcroft R, Shaw J (2011) Telling stories and hearing voices: narrative work with voice hearers in acute care. Journal of Psychiatric and Mental Health Nursing 18: 837-42.

Royal College of Psychiatrists (2011) Do the Right Thing: How to Judge a Good Ward. Ten Standards for Adult In-Patient Mental Healthcare (Occasional Paper OP79). Royal College of Psychiatrists.

Ruddle A, Mason 0, Wykes T (2011) A review of hearing voices groups: evidence and mechanisms of change. Clinical Psychology Review 31: 757-66.

Sackett D, Rosenberg W, Muir Gray J, et al (1996) Evidence based medicine: what it is and what it isn't. BMJ 312: 71.

Shattell M (2007) Boredom in acute psychiatric care. Issues in Mental Health Nursing 28: 661-2.

Simpson A, Bowers L, Alexander J, et al (2005) Occupational therapy and multidisciplinary working on acute psychiatric wards: the Tompkins Acute Ward Study. British Journal of Occupational Therapy 68: $545-52$.

Stahl SM (2008) Stahl's Essential Psychopharmacology: Neuroscientific Basis and Practical Applications. Cambridge.

Thomas N, Pilgrim D, Street C, et al (2012) Supporting young people with mental health problems: lessons from a voluntary sector pilot. Mental Health Review Journal 17: 14-25.

Thomas S, Shattell M, Martin T (2002) What's therapeutic about the therapeutic milieu. Archives of Psychiatric Nursing 16: 99-107.

Todman M (2003) Boredom and psychotic disorders: cognitive and motivational issues. Psychiatry 66: 146-67.

Wise RA (2004) Dopamine, learning and motivation. Nature Reviews Neuroscience 5: 483-94. 
MCQs

Select the single best option for each question stem

1 Alleviating patient boredom in adult acute psychiatric wards is the function of:

a occupational therapists

b nurses

c psychiatrists

d psychologists

e all of the above.

2 Regarding the organisation of activities on psychiatric wards:

a patients need their time to be filled

b generic 'diversionary' activities are likely to address only the external dimensions of boredom

c it does not matter whether or not activities stimulate patients' perceptions of a sense of control

d generic 'diversionary' activities are likely to address only the internal dimensions of boredom

e it does not matter whether activities provide patients with an opportunity to occupy meaningful roles.
3 Which of these statements most accurately describes how antipsychotics may be a significant contributor to boredom?

a Antipsychotics block dopamine, a neurotransmitter which is important for motivation, pleasure and reward.

b Antipsychotics stimulate dopamine, a neurotransmitter which is important for motivation, pleasure and reward.

c It is impossible to say whether antipsychotics could be a boredom contributor because no research studies have separated their effects from the negative symptoms of schizophrenia.

$d$ Risperidone is the antipsychotic which is least likely to be a potential boredom contributor.

e Haloperidol is the antipsychotic which is most likely to be a potential boredom contributor.

4 A successful team approach to boredom might involve:

a using a patient workbook to coordinate a personalised activity plan

b using a graded approach to activities

c regularly reviewing negative effects of medication on motivation and concentration d protecting one-to-one therapeutic time by reducing meetings and administration e all of the above.

5 Which of the following statements best describes the role of a clinical librarian?

a Clinical librarians facilitate access to the evidence base to support clinical decisionmaking and to inform strategy and changes.

b Clinical librarians are exclusively concerned with teaching evidence-based practice skills to health professionals.

c Clinical librarians are well established in uk mental health services.

d Clinical librarians only support the practice of hospital consultants.

e Clinical librarians can only provide evidence summaries to assist with clinical decisionmaking. 